\title{
Functional Recovery from Desensitization of Vanilloid Receptor TRPV1 Requires Resynthesis of Phosphatidylinositol 4,5-Bisphosphate
}

\author{
Beiying Liu, Chunguang Zhang, and Feng Qin \\ Department of Physiology and Biophysical Sciences, State University of New York at Buffalo, Buffalo, New York 14214
}

\begin{abstract}
Capsaicin and other naturally occurring pungent molecules have long been used as topical analgesics to treat a variety of chronic pain conditions. The analgesic effects of these compounds involve long-term desensitization of nociceptors after strong stimulation. To elucidate the underlying mechanisms, we studied the recovery from desensitization of the vanilloid receptor TRPV1. We showed that prolonged applications of capsaicin led to nearly complete desensitization of the channel and that its functional recovery from desensitization required a high concentration of intracellular ATP. Nonhydrolyzable ATP analogs did not substitute for ATP to promote recovery. Neither inhibition nor activation of protein kinases prevented recovery of the channel from desensitization. In contrast, blockade of lipid kinases, in particular phosphatidylinositol-4-kinase, abolished recovery, as did activation of membrane receptors that stimulate hydrolysis of phosphatidylinositol 4,5-biphosphate $\left(\mathrm{PIP}_{2}\right)$. Additional experiments using the $\mathrm{PIP}_{2}$-sensitive inward rectifier potassium channel Kir2.1 as a biosensor showed a high degree of temporal correlation between the two channels on both functional suppression after capsaicin stimulation and subsequent recovery. These data suggest that depletion of $\mathrm{PIP}_{2}$ occurs concomitantly with activation of TRPV1 and its replenishment in the membrane determines recovery of the channel from desensitization. In addition to revealing a new role of phosphoinositide signaling in regulation of nociception, our results provide novel insight into the topical mechanisms of the analgesic effects of capsaicin and the strategies to improve its effectiveness.
\end{abstract}

Key words: capsaicin; analgesic; pain; TRP channels; $\mathrm{PIP}_{2}$; sensory neurons

\section{Introduction}

Responsiveness to capsaicin, the pungent ingredient of hot peppers, is a unique pharmacological trait of a subset of primary afferent neurons (Holzer, 1991). At low suprathreshold doses, locally applied capsaicin stimulates the chemosensitive and thermosensitive nociceptors and elicits pain (Szolcsanyi, 1993). However, at higher doses or with prolonged exposures, it causes desensitization of neurons at both pharmacological and functional levels, resulting in impairment of pain transmission mechanisms (Jancsó, 1968). Systemic capsaicin treatment, in contrast, produces long-term neurotoxicity ranging from ultrastructural damages to degeneration of cells (Jancso et al., 1977; Marsh et al., 1987). The antinociceptive effect of capsaicin on sensory neurons has been exploited for its therapeutic values (Szallasi and Blumberg, 1996).

The molecular target of capsaicin, the vanilloid receptor TRPV1, is a nonselective cation channel of the TRP superfamily (Caterina et al., 1997). The receptor is activated by noxious stimuli such as vanilloids, heat, and acids, and the activity is sensitized

Received Feb. 8, 2005; accepted April 8, 2005.

This work was supported by National Institutes of Health Grants R01-RR11114 and R01-GM65994. We thank P. A. Gottlieb for reading this manuscript.

Correspondence should be addressed to Dr. Feng Qin, State University of New York at Buffalo, 124 Sherman Hall, Buffalo, NY 14214. E-mail: qin@buffalo.edu.

DOI:10.1523/JNEUROSCI.1296-05.2005

Copyright $\odot 2005$ Society for Neuroscience $\quad$ 0270-6474/05/254835-09\$15.00/0 by substances that are present in the inflammatory milieu such as nerve growth factor (NGF), prostaglandins, bradykinin, and lipid metabolites (Julius and Basbaum, 2001). Functional studies of mice lacking TRPV1 support a prominent role for the channel in thermal transduction and pain sensation, especially inflammation-induced thermal hyperalgesia (Caterina et al., 2000; Davis et al., 2000).

The cloned vanilloid receptor TRPV1 explains most of the biological actions of capsaicin on sensory neurons, including its antinociceptive effects. Prolonged or repeated applications of capsaicin cause persistent desensitization of TRPV1 (Caterina et al., 1997). Previous studies in cultured neurons show that the desensitization of capsaicin responses is dependent on a variety of factors, including the capsaicin concentration, the period of stimulus, and the presence of extracellular $\mathrm{Ca}^{2+}$ (for review, see Szallasi and Blumberg, 1999), and can be reduced by inhibition of protein phosphatase 2B (calcineurin) (Docherty et al., 1996). Recent studies of TRPV1 in heterologous expression systems have implicated other mechanisms such as protein kinase A (PKA) phosphorylation and calmodulin (CaM) binding in regulation of desensitization (Bhave et al., 2002; Mohapatra and Nau, 2003; Numazaki et al., 2003; Rosenbaum et al., 2004). Activation of protein kinase $\mathrm{C}(\mathrm{PKC})$, in contrast, is reported to reverse desensitization of capsaicin responses in a $\mathrm{Ca}^{2+}$-imaging assay (Mandadi et al., 2004).

In contrast to the intense studies of desensitization, little is 
known about recovery of TRPV1 from desensitization. The recovery of the channel has been suggested to underlie tachyphylaxis of capsaicin responses, a process that involves successive diminution of responsiveness after brief and repeated capsaicin applications (Liu and Simon, 1996). The molecular mechanisms of the process, however, remain to be elucidated. In this study, we demonstrate that full recovery of TRPV1 from desensitization requires resynthesis of phosphatidylinositol 4,5-biphosphate $\left(\mathrm{PIP}_{2}\right)$ by phosphatidylinositol-4-kinase (PI4K). Inhibition of protein kinases or disruption of the putative phosphorylation sites in the channel does not prevent recovery. Neither does truncation of the distal $\mathrm{C}$ terminus containing a previously identified $\mathrm{PIP}_{2}$-binding region (Prescott and Julius, 2003). The effects of $\mathrm{PIP}_{2}$ on recovery of TRPV1 therefore represent a novel mechanism for phosphoinositide signaling in modulation of TRPV1 activity.

\section{Materials and Methods}

Materials. Capsaicin was obtained from Fluka (Buchs, Switzerland). Protein kinase A inhibitor (PKI) 14-22, forskolin, and 1-oleoyl-2-acetyl-snglycerol (OAG) were purchased from Calbiochem (La Jolla, CA). PMA was obtained from LC Laboratories (Woburn, MA). Capsazepine was purchased from Precision Biochemicals (Vancouver, British Columbia, Canada). All other chemicals, including wortmannin, phenylarsine oxide (PAO), NGF, bisindolylmaleimide I (BIM), N-[2-(p-bromocinnamylamino)ethyl]-5-isoquinolinesulfonamide dihydrochloride (H89), 2-(4morpholinyl)-8-phenyl-4H-1-benzopyran-4-one (LY294002), ATP, $\beta, \gamma$-methyleneadenosine $5^{\prime}$-triphosphate (AMP-PCP), 5'-adenylylimidodiphosphate (AMP-PNP), and $\mathrm{IP}_{3}$, were obtained from Sigma (St. Louis, MO).

Water-insoluble reagents were dissolved in either $100 \%$ ethanol or DMSO to make a stock solution and diluted into a recording solution at the appropriate concentration before the experiment. The final concentrations of ethanol and DMSO did not exceed 0.17 and $0.3 \%$, respectively.

Mutagenesis. Site-directed mutagenesis was performed using the overlap-extension PCR method. Truncations of the $\mathrm{C}$ terminus were done by PCR amplification of primers with the appropriate restriction sites $\left(5^{\prime}\right.$, SacII; $\left.3^{\prime}, X b a \mathrm{I}\right)$. All recombinant constructs were confirmed by restriction enzyme digestion and by DNA sequence analysis.

Heterologous expression. Rat TRPV1 and trkA/p75 (wild type and mutants) were generously provided by David Julius (University of California, San Francisco, CA) (Caterina et al., 1997; Prescott and Julius, 2003). Kir2.1 was provided by Lily Jan (University of California, San Francisco, CA) (Kubo et al., 1993). Human embryonic kidney 293 (HEK293) cells were maintained in DMEM plus 10\% fetal bovine serum (HyClone, Logan, UT) with $1 \%$ penicillin/streptomycin, incubated at $37^{\circ} \mathrm{C}$ in $5 \%$ $\mathrm{CO}_{2}$, and transfected at a confluence of $\sim 80 \%$ using the standard calcium phosphate precipitation method, as described previously (Liu et al., 2004). Either green fluorescent protein or human CD8 lymphocyte antigen $(0.5 \mu \mathrm{g} / 0.2 \mathrm{ml})$ was cotransfected as a surface marker. Electrophysiological recordings took place $10-28 \mathrm{~h}$ after transfection. For cells cotransfected with $\mathrm{CD} 8$, antibody-coated beads were used to visually identify the transfected cells (Dynabeads M450 CD8; Dynal, Lake Success, NY). More than $90 \%$ of the bead-decorated cells appeared to express the channel.

Electrophysiology. Conventional whole-cell recording methods were used. Currents were amplified using either an Axopatch 200B (Molecular Devices, Foster City, CA) or a PC-505B (Warner Instruments, Hamden, CT) patch-clamp amplifier, filtered at $1 \mathrm{kHz}$, and digitized at $5 \mathrm{kHz}$ directly onto the computer hard disk through a BNC-2090/MIO acquisition system (National Instruments, Austin, TX) driven by a customdesigned software $(\mathrm{QuB} / \mathrm{IcE})$. Patch electrodes were fabricated from borosilicate glass (Sutter Instruments, Novato, CA). Drug delivery was controlled by a gravity-driven local perfusion system (ALA Scientific Instruments, Westbury, NY) or intracellular dialysis through a patch pipette for 5-10 min after membrane breakthrough. The recording ap-
A

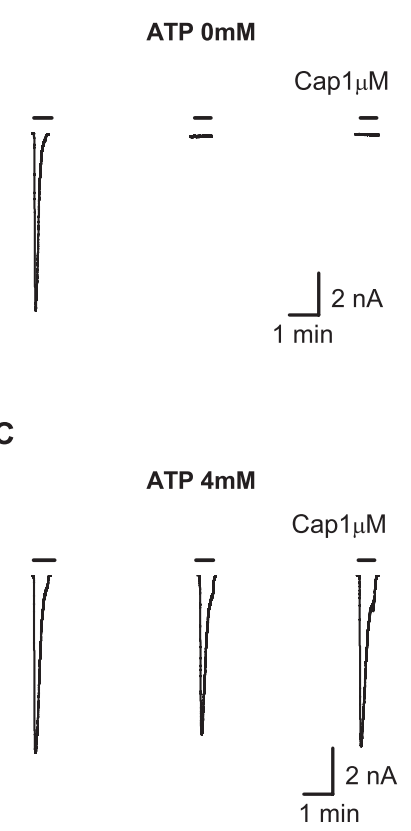

B

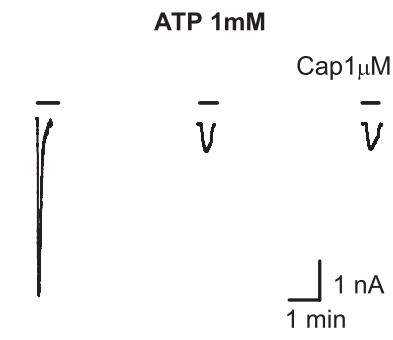

D

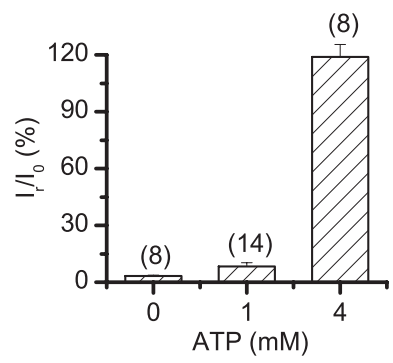

Figure 1. Intracellular ATP promotes recovery of TRPV1 from desensitization. A, Whole-cell recording from a HEK293 cell transiently expressing TRPV1 in response to $1 \mu \mathrm{m}$ capsaicin in the presence of $1.8 \mathrm{~mm} \mathrm{Ca}^{2+}$. The trace includes three repeated applications of capsaicin separated by $\sim 5$ min washes with the standard bath solution. Each capsaicin application lasted for a prolonged duration to ensure full desensitization. The holding potential was $-60 \mathrm{mV}$. B, C, The same experiments as in $A$, except with 1 and $4 \mathrm{~mm} \mathrm{ATP}$ included in the pipette, respectively. $\boldsymbol{D}$, Summary graph of recovery from desensitization plotted as a percentage of the maximum current evoked in response to the second and third applications of capsaicin relative to the peak response to the first application (mean \pm SEM). The number of cells in each trial is indicated in parentheses above each bar. Cap, Capsaicin.

paratus and perfusion lines were always washed thoroughly with ethanol after the experiments. All experiments were conducted at room temperature $\left(22-24^{\circ} \mathrm{C}\right)$.

The control bath solution contained the following (in $\mathrm{mm}$ ): $140 \mathrm{NaCl}$, $5 \mathrm{KCl}, 1.8 \mathrm{CaCl}_{2}, 10 \mathrm{HEPES}$, and 10-30 glucose, pH 7.4 (adjusted with $\mathrm{NaOH}$ ). The standard pipette solution consisted of the following (in $\mathrm{mm}$ ): $140 \mathrm{CsCl}, 1 \mathrm{EGTA}$, and 10 HEPES, pH 7.4 (adjusted with CsOH). For all of the experiments in this study, the pipette solution contained 1 mM EGTA for intracellular $\mathrm{Ca}^{2+}$ buffering. In a subset of experiments, the pipette solution was supplemented with $2 \mathrm{~mm} \mathrm{Mg}^{2+}$. The reagents were generally applied at their supramaximal concentrations (Davies et al., 2000).

\section{Results}

\section{Intracellular ATP confers recovery from desensitization}

We studied recovery of TRPV1 from desensitization in transiently transfected HEK293 cells. Desensitization of the channel was induced by a prolonged exposure to $1 \mu \mathrm{M}$ capsaicin in the presence of $1.8 \mathrm{~mm} \mathrm{Ca}^{2+}$. The agonist was applied until the desensitization of the currents reached a relatively steady-state level. The same stimulus was then repeated in a time interval of $\sim 5 \mathrm{~min}$ for two to three consecutive times. The extent of recovery was quantified as the peak current attainable during the second or third stimulation relative to the peak current evoked by the first application. Figure $1 A$ shows an example of whole-cell recordings obtained with such a protocol. The desensitization of the channel was nearly complete in response to the first application of $1 \mu \mathrm{M}$ capsaicin. The half-decay time of the desensitization was on the order of seconds $\left(t_{1 / 2}, 6 \pm 1 \mathrm{~s} ; n=7\right)$. The experiment 
included no ATP in the pipette solution. Under this condition, there was virtually no recovery of channel activity within the experimental times (5-30 min). This failure of recovery persisted regardless of the washout duration between successive stimuli, indicating that removal of the agonist did not suffice to reverse desensitization. In addition, the loss of the responsiveness was unlikely caused by channel rundown. Dialysis of cells for a prolonged duration before recording did not incur appreciable suppression of the initial responses, whereas shortening capsaicin exposure durations could extend the responsiveness period. The loss of the responsiveness therefore resulted from desensitization of the channel and reflected a failure of recovery.

We explored a role for ATP in recovery of TRPV1 from desensitization. Intracellular nucleotide analogs have been reported to affect tachyphylaxis of capsaicin responses in native neurons (Koplas et al., 1997). Figure $1 D$ summarizes the effects of intracellular ATP on recovery of the channel at different concentrations. When the pipette solution contained $4 \mathrm{~mm} \mathrm{ATP}$, the channel recovered completely over a time course of 5-10 min (Fig. $1 C)$. When the ATP concentration was decreased to $1 \mathrm{~mm}$, the extent of recovery was dramatically diminished (Fig. $1 B$ ) and became only slightly higher than in the absence of ATP (Fig. 1D). The recovery of TRPV 1 from desensitization therefore requires a high concentration of intracellular ATP. In the case of full recovery, the recovered capsaicin responses exhibited rapid acute desensitization with characteristics similar to those of the initial responses. In some patches, the recovery was $>100 \%$, suggesting that either the number of functional channels increased on the plasma membrane or the channel activity was potentiated. The intracellular $\mathrm{Mg}^{2+}$ appeared to facilitate recovery of the channel (data not shown) but was not required. The same experiments with $\mathrm{Na}_{2} \mathrm{ATP}$ produced recovery as strong as with MgATP. The cytosolic ATP is therefore the determinant to promote recovery of the channel from desensitization.

\section{Nonhydrolyzable ATP analogs do not substitute for ATP}

We studied whether hydrolysis of ATP is required for its effects on recovery of TRPV1 from desensitization. Replacement of ATP in the pipette solution with an equimolar amount of the nonhydrolyzable analog AMP-PNP resulted in a nearly complete ablation of recovery (Fig. 2, $B$ vs $A$ ). There remained only small responses to the second and third applications of capsaicin, which had amplitudes similar to the residual currents at the end of the first stimulus, suggesting that they were produced by the remaining nondesensitized channels. Contrary to the pronounced effects on recovery, the nonhydrolyzable analog did not appear to alter desensitization of the channel.

One potential complication with AMP-PNP is that it exists as the lithium salt, and the $\mathrm{Li}^{+}$ions have been reported to affect multiple cellular processes in the millimolar range, including phosphoinositide turnover during stimulation of phospholipase C (PLC)-coupled receptors (Jenkinson et al., 1994; Suh and Hille, 2002). To ensure that $\mathrm{Li}^{+}$was not underlying the apparent effect of AMP-PNP, we tested another nonhydrolyzable analog, AMPPCP, which is a sodium salt. As shown in Figure $2 C$, an equimolar substitution of ATP with AMP-PCP caused a similar failure of recovery. The nonhydrolyzablility of the analogs is therefore responsible for their inability to confer recovery.

Additional experiments were performed on the competitiveness of ATP and its nonhydrolyzable analogs. Whereas a full level of recovery occurred with $4 \mathrm{mM} \mathrm{ATP}$ (Fig. $2 \mathrm{~A}$ ), the addition of an equimolar amount of either AMP-PNP or AMP-PCP in the pipette solution reduced the recovery to only a fractional level (Fig.
A

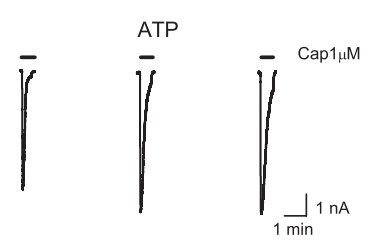

AMP-PNP

$\bar{w}$

$\bar{\imath}$

C

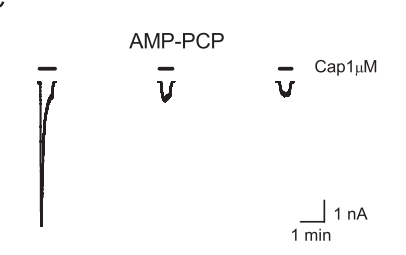

D

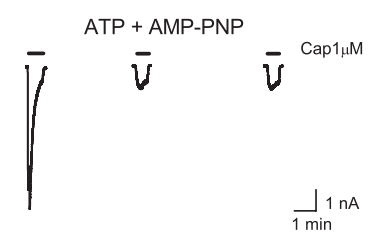

$E$

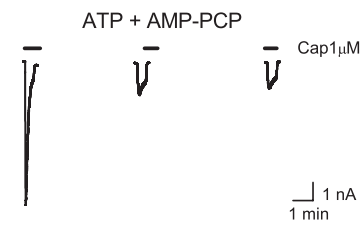

$\mathrm{F}$

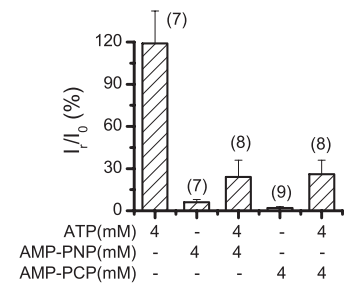

Figure 2. Nonhydrolyzable ATP analogs do not substitute for ATP in recovery. A-E, Wholecell recordings from HEK293 cells transiently expressing TRPV1, showing the recovery from desensitization obtained under different combinations of ATP and its nonhydrolyzable analogs AMP-PNP and AMP-PCP. Currents were elicited by $1 \mu \mathrm{m}$ capsaicin in the presence of $1.8 \mathrm{~mm}$ $\mathrm{Ca}^{2+}$ at $V_{\mathrm{h}}=-60 \mathrm{mV}$. The pipette solution contained the following (in mM): 4 ATP $(\boldsymbol{A}), 4$ $\operatorname{AMP}-P N P(B), 4 \operatorname{AMP}-P C P(C), 4$ ATP plus 4 AMP-PNP $(\boldsymbol{D})$, and $4 \operatorname{ATP}$ plus $4 \operatorname{AMP}-P C P(\boldsymbol{E})$. $\boldsymbol{F}$, Summary of recovery under the various conditions in $\boldsymbol{A}-\boldsymbol{E}$. The number of cells in each trial is indicated in parentheses above each bar. Cap, Capsaicin.

$2 D, E)$ (AMP-PNP: $24 \pm 12 \%, n=8$; AMP-PCP: $26 \pm 10 \%, n=$ $8)$. The nonhydrolyzable analogs therefore counteract the stimulatory effects of ATP, which is consistent with their roles as competitive inhibitors on reactions requiring hydrolyzable ATP. Together, these experiments demonstrated that the hydrolysis of phosphate groups from ATP is essential to recovery of TRPV1 from desensitization.

\section{Protein kinases are not required for recovery}

The need of hydrolyzable ATP for recovery of TRPV1 indicates possible involvement of kinases. Both PKA and PKC have been implicated in desensitization of TRPV1 (Bhave et al., 2002; Mohapatra and Nau, 2003; Mandadi et al., 2004). We therefore explored whether they might mediate recovery of the channel from desensitization. Figure $3 A-D$ summarizes the results on PKA involvement. Several reagents were tested, including two structurally dissimilar inhibitors, H89 and myristoylated PKI, and the adenylate cyclase activator forskolin. As evident from Figure 3, A and $B$, neither inhibitor abolished recovery of capsaicin responses in the presence of $4 \mathrm{~mm}$ ATP. Conversely, activation of PKA with $10 \mu \mathrm{M}$ forskolin in the presence of $1 \mathrm{~mm}$ ATP did not stimulate recovery (Fig. 3C). This failure of recovery was unlikely attributable to an insufficient amount of ATP for PKA phosphorylation, because protein kinase reactions generally require $<1 \mathrm{mM} \mathrm{ATP}$ (Hilgemann, 1997). In addition, forskolin itself appeared to have no effect on recovery. Increasing the intracellular ATP concentration to $4 \mathrm{mM}$, while in the presence of $10 \mu \mathrm{M}$ forskolin, sufficed 
to salvage full recovery as observed in control experiments (Fig. 3D).

Similar experiments were performed to evaluate the involvement of PKC (Fig. $3 E-H)$. BIM, a potent inhibitor of PKC, did not prevent recovery of capsaicin responses from desensitization (Fig. 3E). In some cells, the recovery was less complete than in untreated cells, but overall, the recovery was strong and reached $91 \pm 6 \%$ $(n=9)$ (Fig. $3 F)$. In contrast, PMA, a strong activator of $\mathrm{PKC}$, did not induce any significant recovery over a time course of 5-10 min (Fig. 3G,H). Therefore, either activation or inhibition of PKC produced little effects on recovery of the channel from desensitization. BIM at the micromolar concentration range also inhibits PKA (Bonnington and McNaughton, 2003). Its inability to block the recovery further excludes the involvement of PKA.

We also corroborated our pharmacological results with mutagenesis experiments. TRPV1 contains a number of putative phosphorylation sites, including S6, S116, T144, and T370 on the $\mathrm{N}$ terminus, S502 between S2 and S3, and S774, S800, and S820 on the C terminus. In vitro phosphorylation has confirmed some of these residues capable of PKA and PKC phosphorylation (Bhave et al., 2002; Numazaki et al., 2002). For each putative residue, we constructed the alanine substitution mutant and repeated the corresponding experiments. Figure $4 A-C$ shows the representative traces of recordings from sampled constructs. All mutants were functional and exhibited strong, rapid desensitization in response to prolonged applications of $1 \mu \mathrm{M}$ capsaicin. Furthermore, none of the mutations abolished recovery from desensitization in the presence of $4 \mathrm{~mm}$ ATP (Fig. 4D). Although the exact level of recovery varied among mutants, the variations were secondary. In all cases, the recovery exceeded $80 \%$, indicating that it was not grossly affected. The mutagenesis results therefore provide additional evidence to support that protein kinasedependent mechanisms are not responsible for recovery of TRPV1 from desensitization.

\section{Inhibition of $\mathrm{PIP}_{2}$ synthesis prevents recovery}

We next investigated whether lipid kinases are involved in recovery of TRPV1 from desensitization. Phosphatidylinositol-3kinase (PI3K) and PI4K are responsible for production of phosphoinositides that are important in cell signaling. We examined whether inhibition of these kinases might affect recovery of the channel. PAO, a trivalent arsenical that reacts with vicinal thiols in proteins, inhibits all isoforms of PI4K and blocks production of PIP 2 from phosphatidylinositol (Wiedemann et al., 1996). Figure $5, A$ and $B$, shows that exposure of cells to $30 \mu \mathrm{M}$ PAO profoundly suppressed recovery. The inhibition was relatively slow, taking $>5$ min to reach a maximal effect in some cells. The effect of PAO was mostly reversed by subsequent applications of the sulfhydryl reagent dithiothreitol (DTT), which reacts with PAO to form an inactive complex (Schaefer et al., 1994).

In addition to PI4K, PAO inhibits other enzymes. To assess whether its effect on recovery of TRPV1 results specifically from inhibition of PI4K, we tested another inhibitor, wortmannin. By irreversibly blocking the PI4K activity, wortmannin has been

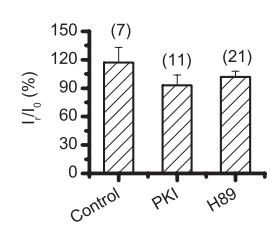

BIM $1 \mu \mathrm{M}$, ATP $4 \mathrm{mM}$
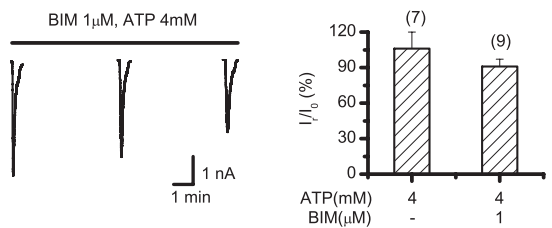

D

G

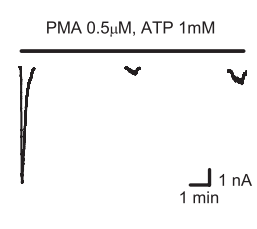

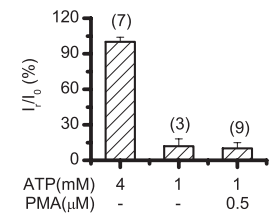

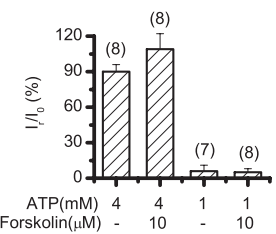

A

Figure 3. Protein kinases do not affect recovery. $\boldsymbol{A}$, Whole-cell currents in response to $1 \mu \mathrm{m}$ capsaicin under inhibition of PKA . controls $(\boldsymbol{F}, \boldsymbol{H})$. In all experiments, the drugs were included in both perfusate and pipette. In a subset of experiments, cells were also exposed to the inhibitors for $\sim 30 \mathrm{~min}$ before experimentation. All recordings were made in HEK293 cells transiently expressing TRPV1 held at $-60 \mathrm{mV}$. The number of cells in each trial is indicated in parentheses above each bar.
A

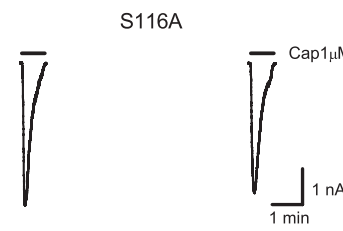

C

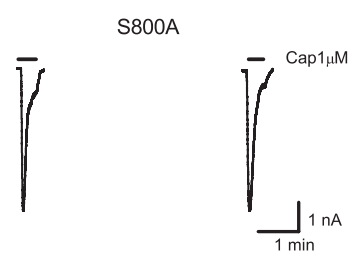

B

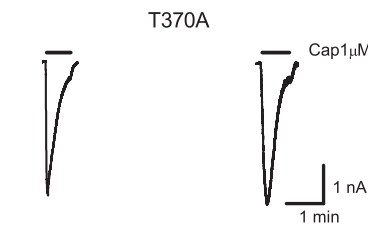

$\mathrm{D}$

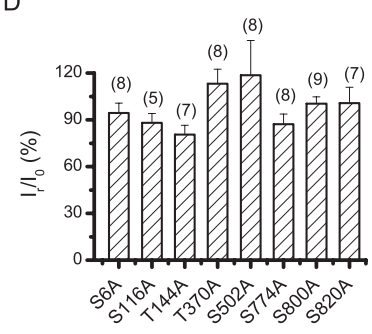

Figure 4. Disruption of putative phosphorylation sites in TRPV1 does not prevent recovery. A-C, Sample recordings of whole-cell responses from HEK293 cells expressing the mutant channels containing alanine substitution at the PKA sites S116 and T370 and the PKC site $\$ 800$. Currents were evoked by consecutive applications of $1 \mu \mathrm{m}$ capsaicin separated with a 5-10 min interval of washes. The pipette solution contained $4 \mathrm{~mm}$ ATP. The holding potential was -60 $\mathrm{mV}$. D, Summary plot of recovery from desensitization of all mutant channels containing a single alanine substitution at each putative phosphorylation site. The number of cells in each trial is indicated in parentheses above each bar. Cap, Capsaicin.

shown to inhibit replenishment of $\mathrm{PIP}_{2}$ in the plasma membrane after receptor-mediated hydrolysis, and its prolonged application depletes $\mathrm{PIP}_{2}$ in unstimulated cells (Varnai and Balla, 1998; Suh and Hille, 2002). Figure 5C-F summarizes the results of wortmannin on recovery of TRPV1 from desensitization. At concentrations above $1 \mu \mathrm{M}$, it exerted an effect similar to PAO, causing a nearly complete inhibition of recovery (Fig. $5 C, D$ ). In contrast, decreasing the concentration to $0.1 \mu \mathrm{M}$ rendered it ineffective 
A

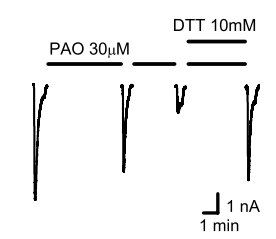

B
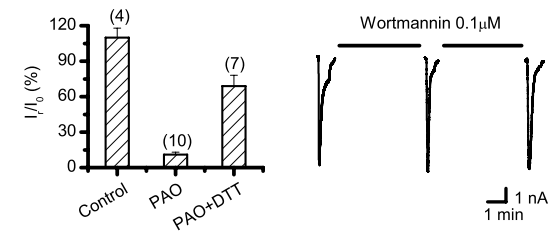

G
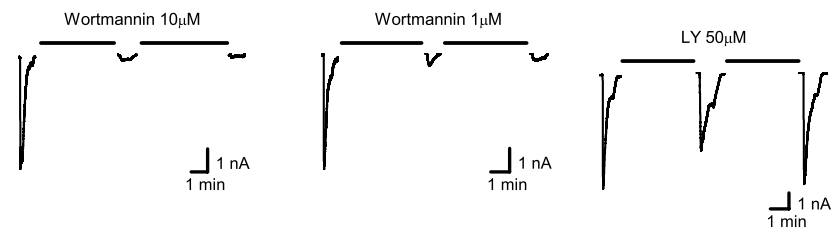

Figure 5. Inhibition of $\mathrm{PIP}_{2}$ synthesis prevents recovery. $\boldsymbol{A}$, Representative recording of whole-cell currents in response to $1 \mu \mathrm{M}$ capsaicin in the presence of PAO. Application of $30 \mu \mathrm{m}$ PAO for 10 min suppressed recovery. The subsequent addition of $10 \mathrm{~mm}$ DTT reversed the effects of PAO. $\boldsymbol{B}$, Averaged plot of recovery under control conditions and applications of PAO in the absence and presence of DTT. $\boldsymbol{C}-\boldsymbol{E}$, Whole-cell recordings from cells treated with 10,1 , and $0.1 \mu \mathrm{m}$ wortmannin, respectively. The recovery of capsaicin responses was inhibited at 1 and $10 \mu \mathrm{m}$ but not at $0.1 \mu \mathrm{M}$. $\boldsymbol{F}$, Summary of the effects of wortmannin (WT) on recovery of capsaicin responses compared with same-day control experiments. G, Current trace obtained with inhibition of PI3K by LY294002 $(\mathrm{LY} ; 50 \mu \mathrm{m})$. $\boldsymbol{H}$, Plot of percentage of recovery with and without application of the inhibitor LY294002. In all cases, the pipette solution contained 4 mM ATP. Data were recorded from HEK293 cells transiently expressing TRPV1 held at $-60 \mathrm{mV}$. The number of cells in each trial is indicated in parentheses above each bar.

A

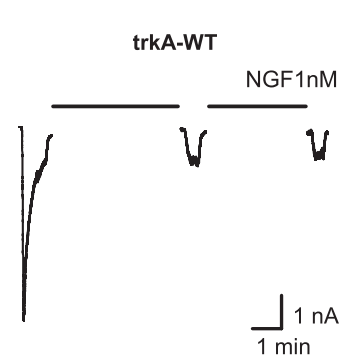

B

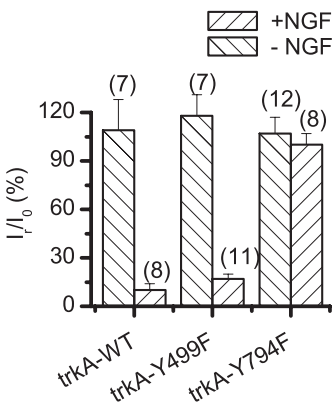

C

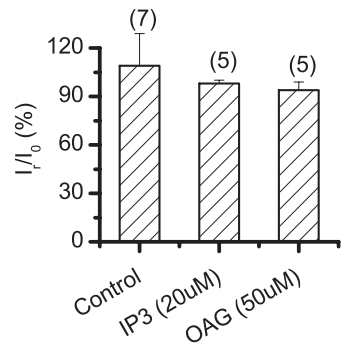

Figure 6. Receptor-mediated hydrolysis of $\mathrm{PIP}_{2}$ inhibits recovery. $\boldsymbol{A}$, Representative recording of whole-cell currents from a HEK293 cell coexpressing TRPV1 and wild-type trkA/p75. NGF (1 nM) was applied between successive capsaicin applications. $\boldsymbol{B}$ Summary plot of percentage of recovery from desensitization for the experiments in $\boldsymbol{A}$ and those involving the mutant trkA receptors $\mathrm{Y499F}$ and $\mathrm{Y794} \mathrm{F}$, with each compared with same-day controls in the absence of NGF. The Y499F and Y794F mutants uncouple the link of trkA to MAPK and PLC- $\gamma$, respectively. C, Summary plot of percentage of recovery for the experiments on the signaling pathways downstream of $\mathrm{PIP}_{2}$ hydrolysis. $\mathrm{IP}_{3}(20 \mu \mathrm{m})$ was dialyzed into cells through the patch pipette, and OAG (50 $\mu \mathrm{M})$, a membrane-permeable analog of DAG, was applied by extracellular perfusion. All data were recorded at $-60 \mathrm{mV}$ with $1 \mu \mathrm{m}$ capsaicin in the presence of $1.8 \mathrm{~mm} \mathrm{Ca}^{2+}$ as the stimulus. The number of cells in each trial is indicated in parentheses above each bar.WT, Wild type.

(Fig. 5E). This concentration dependence of the effect of wortmannin is consistent with the pharmacological profile of the compound as an inhibitor for PI4K. Wortmannin inhibits both $\mathrm{PI} 3 \mathrm{~K}$ and PI4K, but at different concentration ranges (PI3K, 10$100 \mathrm{~nm}$; PI4K, 1-10 $\mu \mathrm{M}$ ) (Davies et al., 2000). The requirement of a relatively high concentration of wortmannin for its effect therefore supports a role for PI4K in recovery of TRPV1 from desensitization.

The involvement of PI4K rather than PI3K was further differentiated with the specific PI3K inhibitor LY294002, which acts via a different mechanism than wortmannin (Vlahos et al., 1994). As seen in Figure 5G, applications of LY294002 at a supramaximal concentration $(50 \mu \mathrm{M})$ did not inhibit recovery of capsaicin responses in the presence of $4 \mathrm{~mm}$ intracellular ATP. Approximately the same size of responses was obtained between the first

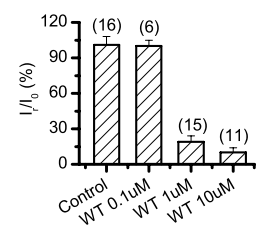

H

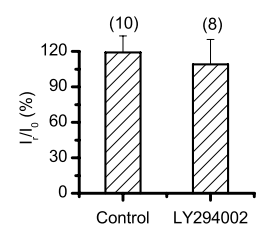

and the subsequent applications of $1 \mu \mathrm{M}$ capsaicin (Fig. 5H). The acute desensitization of the channel was not blocked either. Together, these results suggest that PI4K is specifically required for recovery of TRPV1 from desensitization.

\section{Receptor-mediated hydrolysis of $\mathrm{PIP}_{2}$ prevents recovery}

The $\mathrm{PIP}_{2}$ level in the plasma membrane of many cells can be manipulated through surface receptor-mediated hydrolysis. We asked whether depletion of $\mathrm{PIP}_{2}$ by receptor-mediated hydrolysis might affect recovery of TRPV1. We coexpressed the channel in HEK293 cells with the NGF receptor trkA. Stimulation of trkA by NGF leads to activation of PLC- $\gamma$, which cleaves $\mathrm{PIP}_{2}$ into two second messengers, membrane-bound DAG and soluble $\mathrm{IP}_{3}$. Figure $6 \mathrm{~A}$ shows a representative recording from a cell transiently coexpressing TRPV1 and trkA/p75. The cell was first exposed to $1 \mu \mathrm{M}$ capsaicin to desensitize the channel, followed by $1 \mathrm{nM}$ NGF for $\sim 10$ min to deplete $\mathrm{PIP}_{2}$. As evident from the figure, application of NGF significantly suppressed the subsequent capsaicin responses. On average, only $\sim 10 \%$ recovery occurred over a time course of 5-10 min, as opposed to a full recovery without application of NGF (Fig. 6B).

The trkA receptors are coupled to both mitogen-activated protein kinase (MAPK) and PLC- $\gamma$ (Stephens et al., 1994). To delineate the two pathways, we examined two trkA mutants, Tyr499Phe and Tyr794Phe, which uncouple, respectively, the MAPK and PLC- $\gamma$ pathways from the trkA receptor (Obermeier et al., 1993; Stephens et al., 1994; Prescott and Julius, 2003). As summarized in Figure 6 B, activation of the Tyr499Phe mutant of trkA suppressed recovery of capsaicin responses $(17 \pm 3 \% ; n=11)$. In contrast, activation of the Tyr794Phe mutant produced little effects (100 $\pm 7 \% ; n=8)$ under a similar condition. These results suggest that the PLC- $\gamma$ pathway, not the MAPK pathway, was responsible for the apparent effect of NGF.

To further pinpoint whether depletion of $\mathrm{PIP}_{2}$ itself sufficed to confer inhibition, we tested the downstream products of $\mathrm{PIP}_{2}$ hydrolysis for their possible effects on recovery. Figure $6 C$ summarizes the percentage of recovery from desensitization of capsaicin responses in the presence of $20 \mu \mathrm{M} \mathrm{IP}{ }_{3}$ or $50 \mu \mathrm{M}$ OAG. Neither dialysis of $\mathrm{IP}_{3}$ nor extracellular perfusion of OAG, a membrane-permeable analog of DAG, altered recovery as application of NGF did. DAG is also known to activate PKC; however, our previous experiments involving inhibition of PKC already excluded a contribution from such a pathway. Together, these results indicate that the inhibitory effects of NGF on TRPV1 recovery result directly from breakdown of $\mathrm{PIP}_{2}$ rather than downstream signaling molecules and pathways. 
Dynamic resynthesis of $\mathrm{PIP}_{2}$ is involved in recovery

The slow time course of recovery implies that $\mathrm{PIP}_{2}$ is not only required for but dynamically involved in the process. We hypothesized that $\mathrm{PIP}_{2}$ is depleted during desensitization, presumably because of the ionotropic $\mathrm{Ca}^{2+}$ influx, and that the recovery of the channel from desensitization subsequently requires resynthesis of $\mathrm{PIP}_{2}$ by lipid kinases. To test this hypothesis, we exploited the inward rectifier potassium channel Kir2.1 (Kubo et al., 1993) as a biosensor to monitor the dynamics of $\mathrm{PIP}_{2}$ in the plasma membrane. The Kir2.1 channel is exquisitely sensitive to $\mathrm{PIP}_{2}$ in the membrane (Huang et al., 1998) and has been used successfully to detect $\mathrm{PIP}_{2}$ changes in Drosophila photoreceptors (Hardie et al., 2001).

We coexpressed Kir2.1 and TRPV1 in HEK293 cells and measured the Kir activity before and after desensitization of TRPV1. The Kir channel was activated by exposure to a high- $\mathrm{K}^{+}$solution at a holding potential of $-60 \mathrm{mV}$. As seen in Figure $7 \mathrm{~A}$, large currents were evoked initially by either high- $\mathrm{K}^{+}$or capsaicin $(1$ $\mu \mathrm{M})$. The current profiles of the responses were reminiscent of those of Kir2.1 and TRPV1 when they were expressed alone, suggesting that coexpression of the two channels did not interfere with the function of the other. After desensitization of TRPV1, however, the Kir response was dramatically diminished. Application of capsaicin therefore caused a concomitant suppression of Kir activity, presumably indicating a loss of $\mathrm{PIP}_{2}$ binding. The same stimulus for Kir was then repeated intermittently throughout the course of recovery of TRPV1. The Kir currents gradually recovered and eventually reached a size comparable with that of the initial response. Immediately after recovery of Kir2.1, application of capsaicin also elicited a large response, indicating that TRPV1 recovered concurrently. Figure $7 B$ summarizes the suppression of Kir activity after TRPV1 desensitization and its subsequent recovery compared with that of TRPV1. It was evident that the recovery of the two channels was highly correlated and had a similar time course that presumably reflects the replenishment of $\mathrm{PIP}_{2}$ in the plasma membrane.

\section{$\mathrm{PIP}_{2}$ binding in the distal $\mathrm{C}$ terminus does not mediate recovery}

$\mathrm{PIP}_{2}$ has been identified to interact with a distal C-terminal region of TRPV1, where the binding causes tonic inhibition of channel activity (Prescott and Julius, 2003). We investigated whether the same structural basis underlies the effect of $\mathrm{PIP}_{2}$ on recovery of the channel from desensitization. We constructed two truncation mutants, namely TRPV $1-\Delta 50$ and TRPV1- $\Delta 62$, which lacked the last $50(788-838)$ and $62(776-838)$ residues, respectively. These truncations either partially remove or completely eliminate the $\mathrm{PIP}_{2}$-binding domain on the distal $\mathrm{C}$ terminus, as well as other functional sites in the region such as the CaM-binding domain (767-801) and the phosphorylation sites Ser800 and Ser820 (Bhave et al., 2002; Numazaki et al., 2003; Mandadi et al., 2004). Figure $8 \mathrm{~A}$ shows representative recordings from cells expressing the truncation mutants. Both constructs were functional and retained pronounced acute desensitization in response to prolonged applications of capsaicin in the presence of $1.8 \mathrm{mM} \mathrm{Ca}^{2+}$. More importantly, their recovery from desensi-
A
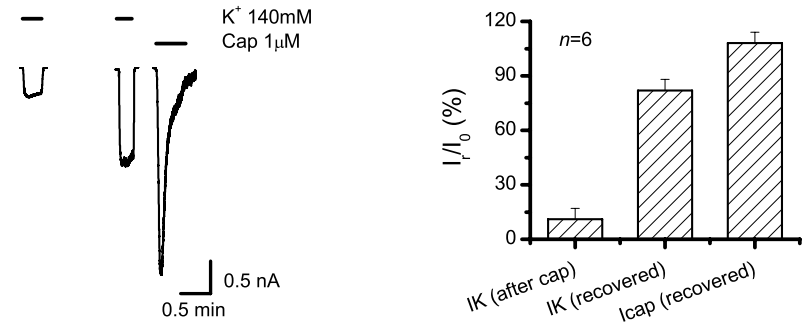

B

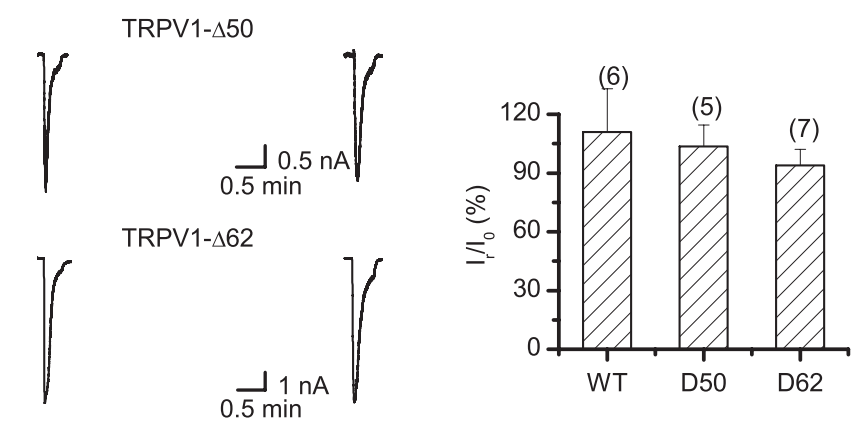

Figure 8. $\mathrm{PIP}_{2}$ binding in the distal $C$ terminus is not important for recovery. $A$, Representative recordings of whole-cell responses to $1 \mu \mathrm{m}$ capsaicin from HEK293 cells expressing the truncation mutants lacking the last 50 (TRPV1- $\Delta 50$ ) and 62 (TRPV1- $\Delta 62$ ) residues, respectively. The holding potential was $-60 \mathrm{mV}$. B, Percentage of recovery of the two truncation mutants versus wild type. The number of cells in each trial is indicated in parentheses above each bar. WT, Wild type.

tization remained strong and had a time course similar to that of wild type. Figure $8 \mathrm{~B}$ shows the averaged recovery of the mutant channels versus wild type. There appeared to be a decreasing trend on the level of recovery with the extent of truncation. But the differences were small, and in all cases, the recovery was robust (TRPV1- $\Delta 62: 94 \pm 8 \%, n=7$; TRPV1- $\Delta 50: 104 \pm 11 \%, n=$ 5 ; wild type, $111 \pm 22 \%, n=6$ ). These data indicate that the $\mathrm{PIP}_{2}$-binding region on the distal $\mathrm{C}$ terminus of TRPV1 is not important for functional recovery of the channel; $\mathrm{PIP}_{2}$ either binds to a different site on the channel or interacts with it indirectly.

\section{Discussion}

Desensitization to prolonged stimuli after activation is a common phenomenon for surface receptors including ion channels. Not the least, the desensitization protects cells from potential excitotoxicity that arises from excessive activation of a receptor. This is particularly the case for $\mathrm{Ca}^{2+}$-permeable ion channels such as TRPV1. The desensitization of TRPV1, however, is unique on several aspects: (1) it depends on extracellular $\mathrm{Ca}^{2+}$; (2) it requires a whole-cell, although not necessarily neuronal, context; and (3) the channel does not immediately recover from desensitization to the resting states after removal of the stimulus. 
Here, we have addressed the problem of recovery of capsaicin responses after desensitization. We showed that the recovery of the channel is conferred by a high concentration of intracellular ATP and is prevented by depletion of $\mathrm{PIP}_{2}$ through either inhibition of $\mathrm{PIP}_{2}$ synthesis kinases or receptor-mediated hydrolysis. We also demonstrated that stimulation of TRPV1 causes concomitant suppression of Kir activity in cells coexpressing the two channels and that the recovery of Kir is concurrent with that of TRPV1. From these results, we conclude that the recovery of TRPV1 from desensitization requires resynthesis of $\mathrm{PIP}_{2}$.

The requirement of $\mathrm{PIP}_{2}$ synthesis for recovery of TRPV1 is consistent with the dependence of the process on a high concentration of intracellular ATP. Whereas protein kinases have a halfmaximal ATP concentration in the range of hundreds of micromolars (Hilgemann, 1997), lipid kinases generally require a significantly higher concentration for maximal activity $(\sim 1 \mathrm{mM})$ (Balla, 1998). The production of phospholipids is also slow and generally takes several minutes, involving a series of steps including phosphorylating phosphatidylinositol lipid on multiple positions (Varnai and Balla, 1998). Both the concentration profile of ATP and the time course of recovery of TRPV1 are in good agreement with those of other reported cellular processes involving resynthesis of $\mathrm{PIP}_{2}$. The recovery of the KCNQ2/KCNQ3 current from muscarinic suppression, for example, requires $3 \mathrm{mM}$ ATP over a time course of many minutes for a maximal level of recovery (Suh and Hille, 2002).

Our study here differs from some existing ones in the use of stimulus protocol. Previous studies on desensitization of TRPV1 apply capsaicin only for a brief time, typically shorter than the half-decay time of desensitization. The same stimulus is repeated successively, and the desensitization is studied in the context of tachyphylaxis of capsaicin responsiveness. With such a protocol, the desensitization of TRPV1 has been found to depend on a number of intracellular components, including ATP (Koplas et al., 1997), calcineurin (Docherty et al., 1996), PKA (Bhave et al., 2002; Mohapatra and Nau, 2003), PKC (Mandadi et al., 2004), and CaM (Numazaki et al., 2003). However, the brief stimuli have limitations on the mechanistic interpretation of data, because tachyphylaxis is a phenomenological process convolving multiple factors including channel activation, acute desensitization, slow desensitization (if any), and recovery. Any change of a single component in the complex process could alter the apparent rate of tachyphylaxis. To alleviate such complications, we used prolonged applications of capsaicin at a relatively high concentration to ensure full desensitization of the channel. With this protocol, we found that activation of PKC did not confer recovery of TRPV1 from desensitization. This observation appears to be inconsistent with a previous report that activation of PKC reverses desensitization of $\mathrm{Ca}^{2+}$ responses in a $\mathrm{Ca}^{2+}$-imaging assay (Mandadi et al., 2004). One possible explanation is that the brief applications of capsaicin in the previous study may have not caused all channels to be desensitized, so that the rise of $\mathrm{Ca}^{2+}$ levels after PKC stimulation could result from the potentiated responses of nondesensitized channels. The differences in the types of experiments may have also contributed to the discrepancy.

$\mathrm{PIP}_{2}$ has been implicated in a variety of cellular processes and regulations of ion channels and membrane proteins (Hilgemann et al., 2001). Ion channels of the TRP superfamily appear to be particularly amenable to modulation by $\mathrm{PIP}_{2}$. The mechanisms whereby $\mathrm{PIP}_{2}$ modulates these channels, however, seem to be disparate. In the case of TRPV1, PIP 2 has been found to exert constitutive inhibition by binding to the distal $\mathrm{C}$ terminus of the channel (Prescott and Julius, 2003). Other TRP channels in the V or $\mathrm{M}$ subfamily, in contrast, appear to require the presence of $\mathrm{PIP}_{2}$ to support their functions. Depletion of $\mathrm{PIP}_{2}$ causes rundown of the spontaneous activity of TRPM7 (Runnels et al., 2002), inhibits activation of TRPM8 (Liu and Qin, 2005), and may contribute to desensitization of TRPM5 (Liu and Liman, 2003). In the TRPC subfamily, the hydrolysis of $\mathrm{PIP}_{2}$ is a major event leading to TRP-TRPL activation for phototransduction in Drosophila melanogaster (Montell, 1999). Regulation of recovery from desensitization as found in this study represents yet another mechanism of $\mathrm{PIP}_{2}$ on modulation of TRP ion channels.

Our results reinforce an emerging paradigm for $\mathrm{PIP}_{2}$ regulation of ion channels in which the $\mathrm{PIP}_{2}$ level in the membrane may be altered by the ionotropic $\mathrm{Ca}^{2+}$ influx through the channels. Ion channels are often regulated by $\mathrm{PIP}_{2}$ through surface receptors. The effect of $\mathrm{PIP}_{2}$ on recovery of TRPV1, however, requires no surface receptor. Instead, activation of TRPV1 itself suffices to deplete $\mathrm{PIP}_{2}$, as manifested in the dynamic changes of the Kir activity after TRPV1 stimulation. The channel therefore acts as both a surrogate surface receptor and a downstream $\mathrm{PIP}_{2}$ target, giving rise to a bidirectional interaction between $\mathrm{PIP}_{2}$ and the regulation of the channel. The $\mathrm{Ca}^{2+}$ entry through the channel is presumably the trigger that initiates the cascade. Ionotropic $\mathrm{Ca}^{2+}$ influx has also been reported to regulate $\mathrm{PIP}_{2}$ levels in Drosophila photoreceptors (Hardie et al., 2001), but with an opposite effect as on TRPV1. In Drosophila photoreceptors, the $\mathrm{Ca}^{2+}$ entry from TRP channels inhibits long-term response inactivation and, as a result, has been suggested to maintain $\mathrm{PIP}_{2}$ levels by inhibiting PLC activity and facilitating $\mathrm{PIP}_{2}$ recycling. In the case of TRPV1, the mechanisms that couple $\mathrm{Ca}^{2+}$ entry to $\mathrm{PIP}_{2}$ depletion remains to be elucidated. To this end, it is noteworthy that both PLC and PI3K have been suggested to colocalize with TRPV1 (Chuang et al., 2001; Stein and Gordon, 2004; Zhu and Oxford, 2004). Their close proximity to the channel would make them ideal candidates in sensing local $\mathrm{Ca}^{2+}$ signals and mediating depletion of $\mathrm{PIP}_{2}$.

The slow, $\mathrm{PIP}_{2}$-dependent recovery of TRPV1 from desensitization provides a mechanistic explanation of tachyphylaxis of capsaicin responses. Previous investigations of sensory neurons suggest that tachyphylaxis stems from a failure of the channel to recover from a desensitization state (Liu and Simon, 1996). Our data provide additional support for this proposition. First, full recovery of capsaicin response is possible under the condition of a high concentration of intracellular ATP. This excludes the existence of a slowly occurring, irreversible desensitization state. Second, prolonged stimulation of TRPV1 causes acute desensitization that is nearly complete and renders the channel irresponsive to subsequent stimuli in the absence of ATP. The acute desensitization itself is therefore irreversible under this condition and may manifest in the form of tachyphylaxis if each application of capsaicin is kept short to give rise to only a partial level of desensitization. Third, recordings in neurons indicate that tachyphylaxis of capsaicin responses can be reduced or prevented by the addition of intracellular nucleotides, suggesting that the two processes of recovery from desensitization and tachyphylaxis share a similar pharmacological profile. In summary, many aspects of tachyphylaxis of capsaicin responses can be explained as a failure of recovery from acute desensitization without invoking an additional, slowly desensitizing state.

The present study also provides new perspectives to understand the physiological modulations of the TRPV1 channel and the roles of phosphoinositides in the pain and inflammation pathways. For example, TRPV1 is essential to inflammatory heat 
hyperalgesia induced by proinflammatory substances such as NGF (Shu and Mendell, 1999). Several mechanisms have been suggested, one involving PLC- $\gamma$ (Chuang et al., 2001) and others implicating PI3K (Bonnington and McNaughton, 2003; Zhuang et al., 2004). Common to these mechanisms is the depletion of $\mathrm{PIP}_{2}$ by either conversion to other phospholipids or hydrolysis into secondary messengers. It is currently understood that activation of these mechanisms potentiates TRPV1 activity and thereby mediates the proinflammatory effect of NGF. The results presented here, however, add another layer of complexity to the actions of NGF and imply that its stimulatory effect may be accompanied with an inhibitory one on the recovery of desensitized channels. NGF is therefore expected to have distinct, opposing effects on the function of TRPV1. To this end, it is interesting to note that a biphasic effect of NGF on capsaicin responses in native neurons has been reported in which stimulation of cells with NGF causes an initial elevation of the current, followed by a progressive decay (Shu and Mendell, 2001). The decrease of the current after prolonged NGF application may have reflected a failure of recovery of the channel from desensitization. Conceivably, such a bimodal role of NGF may provide a dynamic solution to tune its optimal effects without causing overexcitability of cells. Nevertheless, an unequivocal assessment of the effects of $\mathrm{PIP}_{2}$ depletion on recovery of the channel from desensitization and their physiological significance awaits additional confirmation in a neuronal context.

\section{References}

Balla T (1998) Phosphatidylinositol 4-kinases. Biochim Biophys Acta 1436:69-85.

Bhave G, Zhu W, Wang H, Brasier DJ, Oxford GS, Gereau RW (2002) cAMP-dependent protein kinase regulates desensitization of the capsaicin receptor (VR1) by direct phosphorylation. Neuron 35:721-731.

Bonnington JK, McNaughton PA (2003) Signalling pathways involved in the sensitisation of mouse nociceptive neurones by nerve growth factor. J Physiol (Lond) 551:433-446.

Caterina MJ, Schumacher MA, Tominaga M, Rosen TA, Levine JD, Julius D (1997) The capsaicin receptor: a heat-activated ion channel in the pain pathway. Nature 389:816-824.

Caterina MJ, Leffler A, Malmberg AB, Martin WJ, Trafton J, Petersen-Zeitz KR, Koltzenburg M, Basbaum AI, Julius D (2000) Impaired nociception and pain sensation in mice lacking the capsaicin receptor. Science 288:306-313.

Chuang HH, Prescott ED, Kong H, Shields S, Jordt SE, Basbaum AI, Chao MV, Julius D (2001) Bradykinin and nerve growth factor release the capsaicin receptor from PtdIns(4,5)P2-mediated inhibition. Nature 411:957-962.

Davies SP, Reddy H, Caivano M, Cohen P (2000) Specificity and mechanism of action of some commonly used protein kinase inhibitors. Biochem J 351:95-105.

Davis J, Gray J, Gunthorpe M, Hatcher J, Davey P, Overend P, Latcham J, Clapham C, Atkinson K, Rance K, Grau E, Harper A, Pugh P, Rogers D, Bingham S, Randall A, Sheardown S (2000) Abolition of hyperalgesia, but not algesia, in mice lacking VR1. Eur J Neurosci 12:171.

Docherty RJ, Yeats JC, Bevan S, Boddeke HW (1996) Inhibition of calcineurin inhibits the desensitization of capsaicin-evoked currents in cultured dorsal root ganglion neurones from adult rats. Pflügers Arch 431:828-837.

Hardie RC, Raghu P, Moore S, Juusola M, Baines RA, Sweeney ST (2001) Calcium influx via TRP channels is required to maintain PIP2 levels in Drosophila photoreceptors. Neuron 30:149-159.

Hilgemann DW (1997) Cytoplasmic ATP-dependent regulation of ion transporters and channels: mechanisms and messengers. Annu Rev Physiol 59:193-220.

Hilgemann DW, Feng S, Nasuhoglu C (2001) The complex and intriguing lives of PIP2 with ion channels and transporters. Sci STKE 2001:<http:// stke.sciencemag.org/cgi/content/full/OC_sigtrans;2001-111/re19>.

Holzer P (1991) Capsaicin: cellular targets, mechanisms of action, and selectivity for thin sensory neurons. Pharmacol Rev 43:143-201.
Huang CL, Feng S, Hilgemann DW (1998) Direct activation of inward rectifier potassium channels by PIP2 and its stabilization by G $\beta \gamma$. Nature 391:803-806.

Jancso G, Kiraly E, Jancso-Gabor A (1977) Pharmacologically induced selective degeneration of chemosensitive primary sensory neurones. Nature 270:741-743.

Jancsó N (1968) Desensitization with capsaicin and related acylamides as a tool for studying the function of pain receptors. In: Pharmacology of pain(Lim RKS, Armstrong D, Pardo ED, eds), pp 33-55. New York: Pergamon.

Jenkinson S, Nahorski SR, Challiss RA (1994) Disruption by lithium of phosphatidylinositol-4,5-bisphosphate supply and inositol-1,4,5trisphosphate generation in Chinese hamster ovary cells expressing human recombinant $\mathrm{ml}$ muscarinic receptors. Mol Pharmacol 46:1138-1148.

Julius D, Basbaum AI (2001) Molecular mechanisms of nociception. Nature 413:203-210.

Koplas PA, Rosenberg RL, Oxford GS (1997) The role of calcium in the desensitization of capsaicin responses in rat dorsal root ganglion neurons. J Neurosci 17:3525-3537.

Kubo Y, Baldwin TJ, Jan YN, Jan LY (1993) Primary structure and functional expression of a mouse inward rectifier potassium channel. Nature 362:127-133.

Liu B, Ma W, Ryu S, Qin F (2004) Inhibitory modulation of distal C-terminal on protein kinase C-dependent phospho-regulation of rat TRPV1 receptors. J Physiol (Lond) 560:627-638.

Liu BY, Qin F (2005) Functional control of menthol- and cold-sensitive ion channels TRPM8 by PIP 2 . J Neurosci 25:1674-1681.

Liu D, Liman ER (2003) Intracellular $\mathrm{Ca}^{2+}$ and the phospholipid PIP2 regulate the taste transduction ion channel TRPM5. Proc Natl Acad Sci USA 100:15160-15165.

Liu L, Simon SA (1996) Capsaicin-induced currents with distinct desensitization and $\mathrm{Ca}^{2+}$ dependence in rat trigeminal ganglion cells. J Neurophysiol 75:1503-1514.

Mandadi S, Numazaki M, Tominaga M, Bhat MB, Armati PJ, Roufogalis BD (2004) Activation of protein kinase C reverses capsaicin-induced calcium-dependent desensitization of TRPV1 ion channels. Cell Calcium 35:471-478.

Marsh SJ, Stansfeld CE, Brown DA, Davey R, McCarthy D (1987) The mechanism of action of capsaicin on sensory C-type neurons and their axons in vitro. Neuroscience 23:275-289.

Mohapatra DP, Nau C (2003) Desensitization of capsaicin-activated currents in the vanilloid receptor TRPV1 is decreased by the cyclic AMPdependent protein kinase pathway. J Biol Chem 278:50080-50090.

Montell C (1999) Visual transduction in Drosophila. Annu Rev Cell Dev Biol 15:231-268.

Numazaki M, Tominaga T, Toyooka H, Tominaga M (2002) Direct phosphorylation of capsaicin receptor VR1 by PKCe and identification of two target serine residues. J Biol Chem 277:13375-13378.

Numazaki M, Tominaga T, Takeuchi K, Murayama N, Toyooka H, Tominaga M (2003) Structural determinant of TRPV1 desensitization interacts with calmodulin. Proc Natl Acad Sci USA 100:8002-8006.

Obermeier A, Lammers R, Wiesmuller KH, Jung G, Schlessinger J, Ullrich A (1993) Identification of Trk binding sites for SHC and phosphatidylinositol 3'-kinase and formation of a multimeric signaling complex. J Biol Chem 268:22963-22966.

Prescott ED, Julius D (2003) A modular PIP2 binding site as a determinant of capsaicin receptor sensitivity. Science 300:1284-1288.

Rosenbaum T, Gordon-Shaag A, Munari M, Gordon SE (2004) $\mathrm{Ca}^{2+} / \mathrm{cal}-$ modulin modulates TRPV1 activation by capsaicin. J Gen Physiol 123:53-62.

Runnels LW, Yue L, Clapham DE (2002) The TRPM7 channel is inactivated by PIP(2) hydrolysis. Nat Cell Biol 4:329-336.

Schaefer T, Wiedemann C, Gitler C, Burger MM (1994) Effects of arsenicals on the secretory process in chromaffin cells. Ann NY Acad Sci 710:356-367.

Shu X, Mendell LM (1999) Nerve growth factor acutely sensitizes the response of adult rat sensory neurons to capsaicin. Neurosci Lett 274:159-162.

Shu X, Mendell LM (2001) Acute sensitization by NGF of the response of small-diameter sensory neurons to capsaicin. J Neurophysiol 86:2931-2938. 
Stein AT, Gordon SE (2004) Phosphoinositide-3-kinase interacts with the capsaicin receptor, TRPV1, and may play a role in channel sensitization by nerve growth factor. Soc Neurosci Abstr 30:599.3.

Stephens RM, Loeb DM, Copeland TD, Pawson T, Greene LA, Kaplan DR (1994) Trk receptors use redundant signal-transduction pathways involving Shc and Plc-gamma-1 to mediate Ngf responses. Neuron 12:691-705.

Suh BC, Hille B (2002) Recovery from muscarinic modulation of M current channels requires phosphatidylinositol 4,5-bisphosphate synthesis. Neuron 35:507-520.

Szallasi A, Blumberg PM (1996) Vanilloid receptors: new insights enhance potential as a therapeutic target. Pain 68:195-208.

Szallasi A, Blumberg PM (1999) Vanilloid (capsaicin) receptors and mechanisms. Pharmacol Rev 51:159-212.

Szolcsanyi J (1993) Actions of capsaicin on sensory receptors. In: Capsaicin in the study of pain (Wood J, ed), pp 1-26. London: Academic.
Varnai P, Balla T (1998) Visualization of phosphoinositides that bind pleckstrin homology domains: calcium- and agonist-induced dynamic changes and relationship to myo- $\left[{ }^{3} \mathrm{H}\right]$ inositol-labeled phosphoinositide pools. J Cell Biol 143:501-510.

Vlahos CJ, Matter WF, Hui KY, Brown RF (1994) A specific inhibitor of phosphatidylinositol 3-kinase, 2-(4-morpholinyl)-8-phenyl-4H-1benzopyran-4-one (LY294002). J Biol Chem 269:5241-5248.

Wiedemann C, Schafer T, Burger MM (1996) Chromaffin granuleassociated phosphatidylinositol 4-kinase activity is required for stimulated secretion. EMBO J 15:2094-2101.

Zhu W, Oxford GS (2004) Map-kinase and PI-3-kinase pathways are involved in acute sensitization of TRPV1 by NGF. Soc Neurosci Abstr 30:744.8.

Zhuang ZY, Xu H, Clapham DE, Ji RR (2004) Phosphatidylinositol3-kinase activates ERK in primary sensory neurons and mediates inflammatory heat hyperalgesia through TRPV1 sensitization. J Neurosci 24:8300-8309. 